\title{
European experience of the convergent atrial fibrillation procedure: Multicenter outcomes in consecutive patients
}

\author{
Borut Geršak, MD, PhD, ${ }^{\text {a }}$ Michael O. Zembala, $\mathrm{MD}, \mathrm{PhD},{ }^{\mathrm{b}}$ Dirk Müller, $\mathrm{MD}, \mathrm{PhD},{ }^{\mathrm{c}}$ \\ Thierry Folliguet, MD, PhD, ${ }^{\mathrm{d}}$ Matevz Jan, MD, PhD, ${ }^{\text {a }}$ Oskar Kowalski, MD, PhD, ${ }^{\mathrm{b}}$ Stefan Erler, MD, ${ }^{\mathrm{c}}$ \\ Clement Bars, MD, ${ }^{\mathrm{d}}$ Boris Robic, $\mathrm{MD},{ }^{\mathrm{a}}$ Krzysztof Filipiak, $\mathrm{MD},{ }^{\mathrm{b}}$ and Gerhard Wimmer-Greinecker, MD, $\mathrm{PhD}{ }^{\mathrm{c}}$
}

\begin{abstract}
Background: The objective of this collaborative, multicenter, European effort was to evaluate the outcomes of the convergent procedure for the treatment of persistent and long-standing persistent atrial fibrillation (AF) in consecutive patients at 4 European centers.
\end{abstract}

\begin{abstract}
Methods: Outcomes of consecutive patients, undergoing the convergent procedure at 4 European centers, were evaluated in this study. Epicardial ablation was performed before endocardial ablation. Convergent procedure outcomes were recorded by interrogation of implanted loop recorders or Holter monitors. Rhythm status and required interventions (antiarrhythmic drugs, cardioversions, and repeat ablations) were quantified 6 and 12 months after the procedure. Outcomes, monitoring type, and patient baseline characteristics were analyzed and reported.
\end{abstract}

\begin{abstract}
Results: Seventy-three consecutive patients presenting with persistent AF (30.1\%) or long-standing persistent AF $(69.9 \%$ ) underwent the convergent procedure between January 2010 and December 2011. At 6 months, 82\% $(56 / 68)$ were in sinus rhythm. At 12 months, $80 \%(53 / 66)$ were in sinus rhythm; single-procedure maintenance of sinus rhythm without postblanking period interventions was $76 \%(50 / 66) ; 52 \%(34 / 66)$ were in sinus rhythm and not receiving antiarrhythmic drugs.
\end{abstract}

Conclusions: This multicenter European collaborative effort demonstrated that the convergent procedure is a safe and efficacious treatment option for persistent and long-standing persistent AF. (J Thorac Cardiovasc Surg 2014;147:1411-6)

\begin{abstract}
Atrial fibrillation (AF) treatment needs new modalities to treat the most prevalent and complex forms of AF, persistent and long-standing persistent. Catheter ablation has become a primary treatment modality for drug refractory, symptomatic paroxysmal AF, but has demonstrated limited efficacy in persistent and long-standing persistent AF. The 5-year arrhythmia-free survival for a single catheter ablation procedure in a predominantly paroxysmal patient population has been reported at $29 \%$, whereas the efficacy after multiple procedures was $63 \% .{ }^{1}$ Consequently, the incidence and recurrence of AF greatly exceed the number of patients successfully treated, causing explosive growth and a burden to health care systems around the world. ${ }^{2}$
\end{abstract}

\footnotetext{
From the Department of Cardiovascular Surgery, ${ }^{\text {a }}$ University Medical Center Ljubljana, Ljubljana, Slovenia; the Silesian Center For Heart Diseases, ${ }^{\mathrm{b}}$ Zabrze, Poland; the Herz-und Gefäßzentrum, ${ }^{\mathrm{c}}$ Bad Bevensen, Germany; and L'Institut Mutualiste Montsouris, ${ }^{\mathrm{d}}$ Paris, France.

Disclosures: Drs Gersak, Zembala, and Folliguet are consultants for nContact, Inc. The other authors have nothing to disclose with regard to commercial support.

Received for publication Feb 7, 2013; revisions received June 10, 2013; accepted for publication June 27, 2013; available ahead of print Aug 28, 2013.

Address for reprints: Borut Geršak, MD, PhD, Department of Cardiovascular Surgery, University Medical Center Ljubljana, Zaloska 7, 1000 Ljubljana, Slovenia (E-mail: bgersak@maat.si).

$0022-5223 / \$ 36.00$

Copyright (c) 2014 by The American Association for Thoracic Surgery

http://dx.doi.org/10.1016/j.jtcvs.2013.06.057
}

Isolation of focal triggers associated with the pulmonary veins $(\mathrm{PVs})$ is a cornerstone of any $\mathrm{AF}$ treatment strategy. ${ }^{3,4}$ However, simple PV isolation does not address reentrant circuits associated with persistent and long-standing persistent $\mathrm{AF}$ or patients with structural heart disease and enlarged atria. ${ }^{5}$ The posterior left atrium has become a target of interest in patients having chronic atrial stretch and AF because of observations of conduction abnormalities, including slower conduction, heterogeneity, and numerous lines of functional block. ${ }^{6}$ Therefore, to treat the most prevalent complex forms of $\mathrm{AF}$, an effective procedure must isolate the PVs and the posterior left atrium without hindering heart function or the ability of the heart to reverse remodel once sinus rhythm is restored.

The convergent procedure was developed to directly access and visualize the posterior left atrium to isolate the PVs and ablate the posterior left atrium while offering minimal surgical trauma combined with precision of the electrophysiologic 3-dimensional mapping. The multidisciplinary approach leverages the ability of the endoscopic surgeon to complete lines of conduction block with the electrophysiologist's ability to map the lines of block, identify breakthroughs, and complete isolation of the PVs. By combining these 2 disciplines, a comprehensive pattern of endocardial and epicardial lesions is created without chest incisions, lung deflation, or invasive heart 


\section{Abbreviations and Acronyms \\ $\mathrm{AF}=$ atrial fibrillation \\ $\mathrm{PV}=$ pulmonary vein}

dissections. The purpose of this article is to evaluate outcomes of the multidisciplinary closed chest convergent procedure for the treatment of persistent and longstanding persistent $\mathrm{AF}$.

\section{METHODS}

This is an outcomes data evaluation study performed at 4 European centers in compliance with the Declaration of Helsinki. Local medical ethics committee approval was obtained by each site before study initiation. All patients with a history of persistent or long-standing persistent AF consenting to study participation were eligible for inclusion in the study. Seventy-three patients consented; consecutive AF patients underwent the convergent procedure at the 4 centers between January 2010 and December 2011. A 3-month blanking period was allowed; any $\mathrm{AF}$ treatments within this period were not considered efficacy failures, except any endocardial catheter ablations were recorded as redos.

\section{Multidisciplinary AF Treatment}

The epicardial and endocardial ablation components of the convergent procedure were performed as a single-setting procedure on the same day or as a staged procedure, with the epicardial and endocardial components performed on 2 different days, as 2 different hospitalizations. At one center, all the procedures were performed as staged procedures minimally 2 weeks apart to accommodate local reimbursement challenges. At a second center, $50 \%$ of the procedures were performed as staged procedures minimally 2 months apart.

\section{Transdiaphragmatic Access and Epicardial Ablation}

Endoscopic access to posterior cardiac structures was established through 3 small abdominal stab incisions. Low-pressure insufflation of the belly provided visualization of the central tendon portion of the diaphragm. Epicardial access was first obtained by creating a pericardial window with a shielded Teflon monopolar electrocautery hook probe (ATC Technologies, Wilmington, Mass) through the visualized central tendon of the diaphragm. ${ }^{7}$ A transdiaphragmatic window to the pericardium allowed introduction of a soft shallow angle cannula (nContact, Inc, Morrisville, NC) into a retrocardiac position, giving a direct view of the posterior left atrium and allowing manipulation of the cannula without compromising hemodynamics. After accessing the left atrium, linear epicardial lesions were created along the posterior region of the left atrium. ${ }^{8}$ At all 4 sites, preset power $(30 \mathrm{~W})$ and time (90 seconds) settings for the Numeris Guided Coagulation System (nContact, Inc) were used for the creation of each epicardial lesion.

Figure 1, A, shows the base lesion pattern. Posterior left atrial lesions were created between the left and right pulmonary veins within the oblique sinus that is outlined by the pericardial reflections. After ablating the posterior left atrium between the left and right pulmonary veins, linear lesions were created anterior to the left pulmonary veins toward the ligament of Marshall. Finally, lesions were created anterior to the right pulmonary veins and inferior vena cava parallel to the interatrial groove. After the creation of all epicardial lesions, the abdominal access sites were closed and the patient was prepared for standard left-sided endocardial ablation for the convergent procedures performed in a single setting.

\section{Epicardial Ablation System}

The Numeris Guided Coagulation System, designed for such closedchest, thoracoscopic procedures, was used for the endoscopic visualization of the pericardial space and the creation of epicardial linear lesions. The epicardial ablation device and cannula used to access the pericardial space are shown in Figure 1, $B$ and $C$.

Suction applied to the helical coil electrode ensures consistent and predictable contact with cardiac tissue during radio frequency ablation. A perfusion lumen coupled to the vacuum lumen is routed to an unpressurized bag of saline. Once a vacuum seal is obtained, saline is pulled along the device, cooling the surface of the instrument not in contact with the epicardium and focusing RF energy into the epicardium. This ensures a complete lesion that extends to the endocardium. The vacuum-controlled saline perfusion also provides a visible indicator that a vacuum seal has been maintained.

The design of the device solves the critical requirements for the creation of complete, nonconductive lesions capable of interrupting erratic electrical signals, as demonstrated by chronic good clinical practice preclinical studies. ${ }^{9}$ The lesions are visible along the epicardial surface of the heart, allowing them to be easily connected under direct visualization into a pattern capable of interrupting and silencing deviant circuits. The radiofrequency generator uses an algorithm based on impedance that regulates power to prevent tissue overheating and subsequent vaporization.

The Numeris system has received the Conformité Européenne mark of approval for the coagulation of cardiac tissue using radiofrequency energy for the treatment of arrhythmias, including atrial fibrillation or atrial flutter.

\section{Transseptal Access and Endocardial Catheter Ablation}

Percutaneous venous catheterization was used to introduce catheters into the right atrium. A coronary sinus catheter was inserted to provide a reference for 3D navigation. A transseptal puncture was used to access the left atrium and introduce mapping and ablation catheters. Electrophysiologist mapping techniques, including interrogation of intracardiac electrograms with a Lasso catheter or voltage mapping with Carto3 (Biosense Webster, Diamond Bar, Calif) or NavX (St Jude Medical, St Paul, Minn), were performed to identify breakthroughs between epicardial lesions along the pericardial reflections. Endocardial lesions were created to connect the epicardial lesions and complete the pulmonary vein isolation. Each site used the endocardial catheter and mapping systems of its choice. In Slovenia, Celsius catheters were used without a navigation system, and with power set at 25 to $30 \mathrm{~W}$ and an irrigation rate of $60 \mathrm{~mL} /$ min when ablating and $2 \mathrm{~mL} / \mathrm{min}$ when on standby. In Germany, Thermocool catheters (Biosense Webster) were used with either Carto or NavX mapping systems; endocardial ablation was performed at 20 to $30 \mathrm{~W}$ with an irrigation rate of $17 \mathrm{~mL} / \mathrm{min}$. In France, the Biosense Navistar irrigated catheter was used with the Carto mapping system; during endocardial RF ablation, power settings of 26 to $35 \mathrm{~W}$ were used, with $42^{\circ} \mathrm{C}$ as a target temperature and an irrigation rate of 17 and $22 \mathrm{~mL} / \mathrm{min}$. In Poland, the NaviStar catheter system (Biosense Webster) was used with the Carto 3-dimensional mapping system; endocardial ablation was performed with a power setting of $40 \mathrm{~W}$ for 60 seconds.

All sites performed entrance and/or exit block testing to ensure electrical isolation of the PVs and the posterior atria.

\section{Postoperative Management}

Antiarrhythmic drug therapy was directed by the referring physician throughout follow-up. Anticoagulation was reinitiated postoperatively and continued for at least 3 months, and was also directed by the referring physician. Postoperative pain management was at each physician's discretion. Level of pain was a subjective patient response from low to moderate levels. Hospital stay averaged 5 days for patients undergoing the convergent procedure in a single setting and the epicardial ablation procedure in the staged procedure setting. Hospital stay averaged 2 days for patients undergoing the endocardial ablation procedure in the staged procedure setting.

\section{Follow-up Monitoring}

A total of $65 \%(48 / 73)$ of patients were implanted with implantable loop recorders (Reveal XT Insertable Cardiac Monitors; 


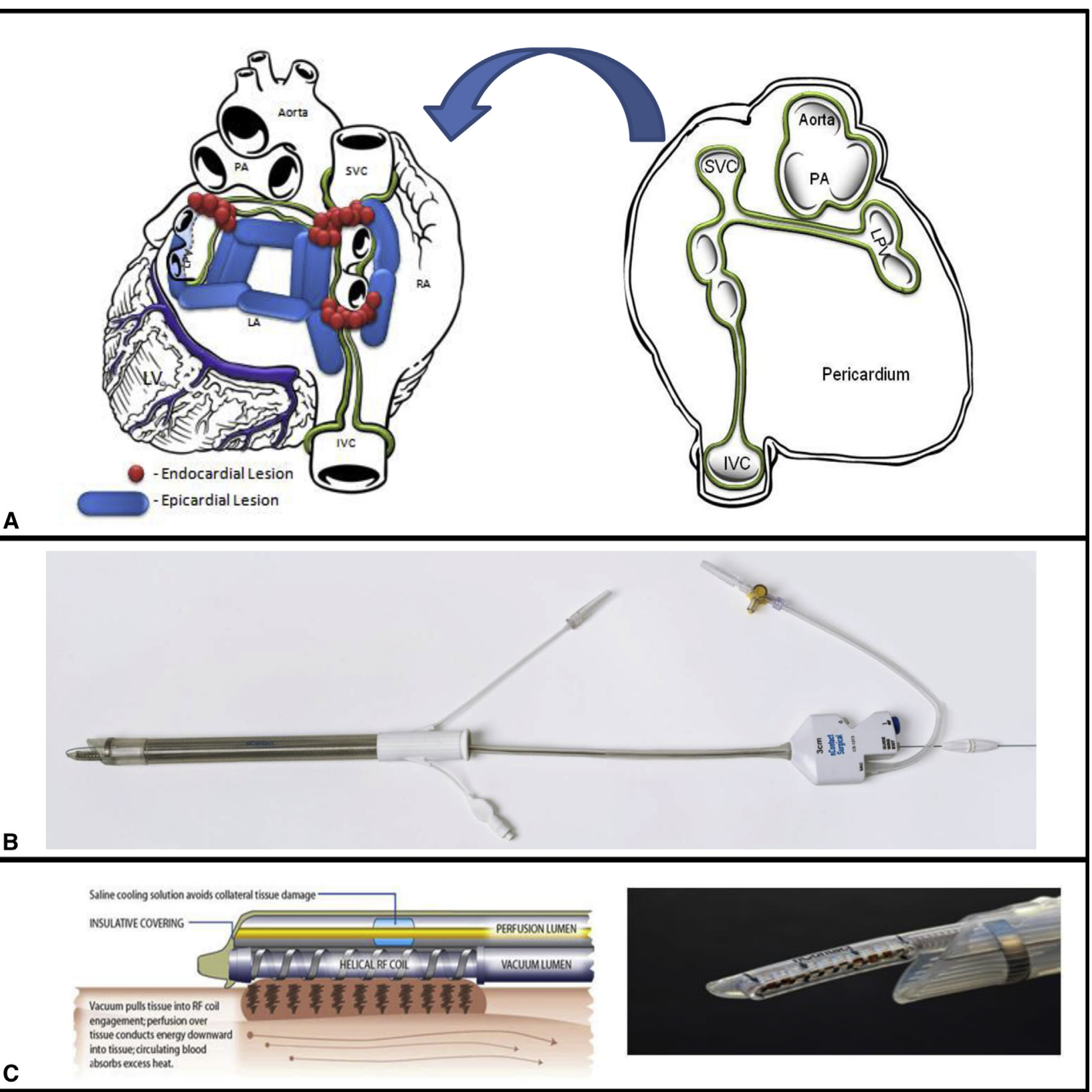

FIGURE 1. A, Base lesion pattern. B, Epicardial coagulation device with cannula. C, Cross-section and close-up of the epicardial coagulation device electrode. $S V C$, Superior vena cava; $R A$, right atrium; $I V C$, inferior vena cava; $L A$, left atrium; $L P V$, left pulmonary vein; $P A$, pulmonary artery; $R F$, radio frequency.

Medtronic, Inc, Minneapolis, Minn). Holter monitoring (24 hours or 7 days) was performed on the remaining patients. Rhythm data were interrogated at regular follow-up intervals (3, 6, and 12 months). Patients were further evaluated with transthoracic echocardiography at 6- and 12-month follow-up visits. Follow-up time points were calculated after the completion of both epicardial and endocardial components of the convergent procedure when performed as a staged procedure.

Follow-up data of 6 months or greater were recorded for 68 patients; of these patients, 66 had 12-month follow-up monitoring data. Two patients who were converted to sternotomies and received epicardial ablation only were excluded from the efficacy analysis.

\section{Statistical Analysis}

Baseline and follow-up values are reported as mean $\pm \mathrm{SD}$ for numeric measures and counts and percentages for categorical measures. KaplanMeier survival analysis was performed for the total patient population. For comparison of left atrial size and left ventricular ejection fraction, paired $t$-tests were performed, with means and SDs reported only for subsets in which data were available at both baseline and 12-month follow-up.

\section{RESULTS}

Seventy-three consecutive patients receiving the convergent procedure were included in the baseline demographic 
data analysis. All patients were persistent or long-standing persistent, with an average $\mathrm{AF}$ duration of $4.3 \pm 4.4$ years, as defined by the Heart Rhythm Society recommendations. ${ }^{10}$ Baseline demographics are detailed in Table 1. The mean patient age was 56.3 years, and $81 \%$ were male. The mean baseline left atrial size and left ventricular ejection fraction were $46.5 \mathrm{~mm}$ and $54.7 \%$, respectively, based on transthoracic echocardiographic measurements.

The skin-to-skin epicardial procedure time was $111.6 \pm$ 38.4 minutes. An average of 19 epicardial lesions were created for each patient, with an epicardial RF ablation time of $22.7 \pm 7.9$ minutes.

Serious adverse event rates are listed in Table 2. There was no operative, perioperative, or late mortality. One cerebral vascular incident was observed. Two patients had bleeding that required conversion to sternotomies. Two more patients had abdominal bleeding that required blood transfusion without intervention.

Efficacy outcomes are detailed in Table 3. A total of $82 \%$ of patients were in sinus rhythm at 6-month follow-up; $76 \%$ were in sinus rhythm without repeat ablation procedures or interventions outside the blanking period. A total of $80 \%$ of patients were in sinus rhythm at 12 months; $76 \%$ had a single-procedure efficacy without subsequent interventions, and $52 \%$ were in sinus rhythm

TABLE 1. Demographic characteristics

\begin{tabular}{lc}
\hline \multicolumn{1}{c}{ Characteristic } & All subjects $(\mathbf{n}=\mathbf{7 3})$ \\
\hline Male, No. (\%) & $59(81)$ \\
Age, y & \\
Mean (SD) & $56.3(10.5)$ \\
$\quad$ Range & $28-75$ \\
BMI, kg/m ${ }^{2}$ & \\
Mean (SD) & $29.4(4.7)$ \\
Range & $20.2-49.1$ \\
Preoperative LA, mm & \\
Mean (SD) & $46.5(5.5)$ \\
Range & $28-57$ \\
Preoperative LVEF, \% & \\
Mean (SD) & $54.7(12.2)$ \\
Range & $20-80$ \\
Atrial fibrillation type, No. (\%) & \\
Persistent & $22(30)$ \\
Long-standing persistent & $51(70)$ \\
AF duration, y & \\
Mean (SD) & $4.3(4.4)$ \\
Range & $0.5-25$ \\
Preexisting conditions, No. (\%) & \\
Congestive heart failure & $16(22)$ \\
Hypertension & $50(68)$ \\
Diabetes & $9(12)$ \\
Stroke/TIA/thromboembolic event & $5(7)$ \\
Vascular disease (CAD, PAD) & $5(7)$ \\
\hline$B M I$, Body mass index; $L A$, left atrium; $L V E F$, left ventricular ejection fraction; \\
$A F$, atrial fibrillation; $T I A$, transient ischemic attack; $C A D$, coronary artery disease; \\
$P A D$, peripheral arterial disease; $S D$, standard deviation. & \\
&
\end{tabular}

TABLE 2. Serious adverse event rates

\begin{tabular}{lc}
\hline \multicolumn{1}{c}{ Serious adverse event } & No. $(\%)$ of $\mathbf{7 3}$ \\
\hline Operative mortality & $0(0)$ \\
$<30-d$ Mortality & $0(0)$ \\
$>30-d$ Mortality & $0(0)$ \\
Stroke/CVA & $1(1.4)$ \\
TIA & $0(0)$ \\
Tamponade & $1(1.4)$ \\
Pericardial effusion & $1(1.4)$ \\
Pleural effusion & $1(1.4)$ \\
Phrenic nerve palsy & $0(0)$ \\
Esophageal fistula & $0(0)$ \\
Myocardial infarction & $0(0)$ \\
Newly developed third-degree AV block & $0(0)$ \\
Acute limb ischemia & $0(0)$ \\
Bleeding requiring transfusion & $2(2.7)$ \\
Bleeding with conversion to sternotomy & $2(2.7)$ \\
\hline
\end{tabular}

Serious adverse events were observed in 8 patients $(8$ of $73 ; 11 \%)$. CVA, Cerebrovascular accident; TIA, transient ischemic attack; $A V$, atrioventricular.

and not taking antiarrhythmic drugs. The 2 patients who were converted to sternotomies and received epicardial ablation only (not included in efficacy analysis) were in $\mathrm{SR}$ at 6 months, with 1 of the 2 patients reverting to AF at 12 months.

Figure 2 shows an arrhythmia- and intervention-free survival analysis throughout 1 year of follow-up. The cumulative survival in terms of lack of arrhythmia recurrence and interventions throughout 1-year follow-up was $73 \%$. Only 3 patients (4\%) required repeat ablation procedures.

Left atrial size decreased throughout follow-up, from an average of $46.5 \pm 5.5 \mathrm{~mm}$ at baseline to $42.9 \pm 6.2 \mathrm{~mm}$ at 12 months $(P<.01)$. Left ventricular ejection fraction increased throughout follow-up, from an average of $54.7 \% \pm 12.2 \%$ at baseline to $58.5 \% \pm 12.6 \%$ at 12 months $(P<.01)$.

\section{DISCUSSION}

Stand-alone surgical and catheter ablation approaches have been limited in the ability to effectively ablate the posterior left atrium or create linear lesions capable of interrupting macro-reentrant circuits. The original cut-and-sew Maze III procedure used a highly invasive,

TABLE 3. Outcomes at each follow-up visit

\begin{tabular}{lc}
\hline Clinical measure/follow-up & $\%$ (No./total) responders \\
\hline Sinus rhythm & \\
6 mo & $82(56 / 68)$ \\
12 mo & $80(53 / 66)$ \\
Sinus rhythm, no interventions & \\
6 mo & $76(52 / 68)$ \\
12 mo & $76(50 / 66)$ \\
\hline
\end{tabular}




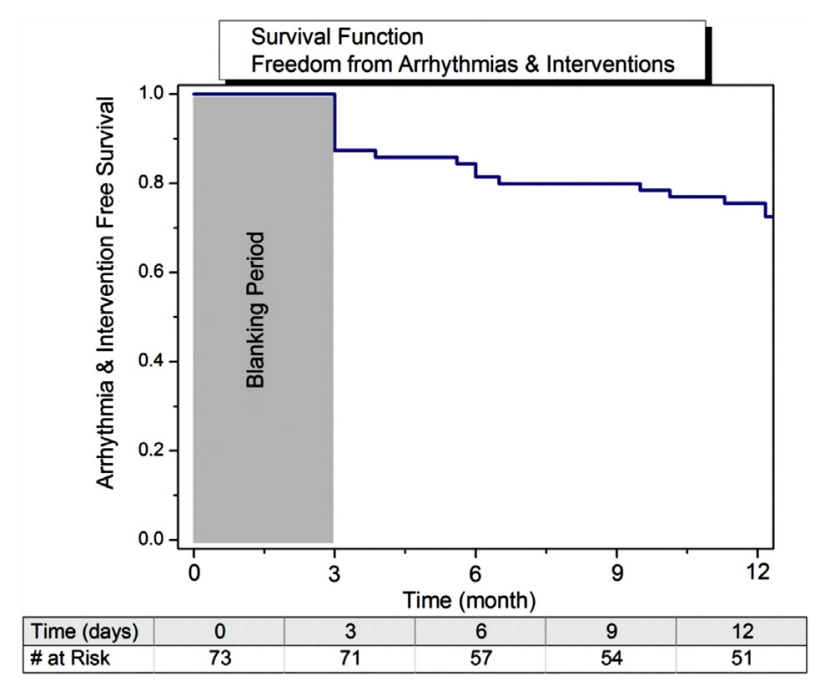

FIGURE 2. Arrhythmia and intervention-free survival analysis.

open-chest approach to isolate the pulmonary veins and posterior left atrium, along with other lines throughout the right and left atria. ${ }^{11}$

Literature suggests the posterior left atrium, bounded by the pericardial reflections and extending between the left and right pulmonary veins, derives embryologically from pulmonary vein tissue and produces lines of functional conduction block that may exacerbate the potential for developing arrhythmogenic substrates. ${ }^{6}$ Access to and visualization of the posterior left atrium may be essential to localize ablation along the target posterior left atria that is tethered by the pericardial reflections.

The convergent procedure combines 2 important medical disciplines for the treatment of persistent and long-standing persistent AF. Laparoscopic epicardial creation of linear lesions is augmented by endocardial catheter ablation along the pericardial reflections; endocardial mapping further ensures pulmonary vein isolation is obtained and the lesion pattern is complete.

This multicenter evaluation of the convergent procedure demonstrated the ability to restore sinus rhythm in persistent and long-standing persistent AF patients and avoid dependence on continued interventions, including cardioversions or repeat catheter ablation procedures. Discontinuation of antiarrhythmic drug therapy is affected by patient choice, physician discretion, and the need for drugs to address other concomitant medical conditions. Despite treating a more difficult AF patient population, the outcomes compare favorably to those published for catheter ablation of paroxysmal AF. ${ }^{12,13}$

Catheter ablation studies have observed a continued decrease in outcomes more than 1 year after AF ablation and require a high redo rate to achieve outcomes greater than $50 \% .^{1,4,14}$ In addition, surgical studies have demonstrated a substantial reduction in outcomes when access limitations inhibit the ability to isolate the posterior left atrium, even when using limited monitoring methods, such as electrocardiograms. ${ }^{15}$

Stand-alone surgical ablation approaches to treat atrial fibrillation have been limited by the morbidity associated with surgical access requiring chest incisions or ports. Based on the Society of Thoracic Surgeons Database, major complications for stand-alone surgical AF ablation occurred in $27.97 \%$ of on-pump and $13.60 \%$ of offpump patients, with an operative mortality of $1.7 \%$ and $0.5 \%$, respectively. ${ }^{16}$ In addition, the high readmission rates $(11 \%)$ highlight the invasiveness of on- and offpump surgical ablation approaches, which will continue to limit widespread adoption compared with catheter ablation.

In comparing catheter ablation with surgical ablation, the FAST randomized study reported 12-month postprocedure freedom from $\mathrm{AF}$ of $65.6 \%$ for surgical ablation and $36.5 \%$ for catheter ablation. However, the procedural adverse events were much higher in the surgical ablation arm, although a comparable number of serious adverse events were reported in both arms during the 12-month follow-up. ${ }^{17}$

The convergent procedure avoids chest incisions and single-lung ventilation to reduce complications associated with surgical ablation while creating a comprehensive lesion pattern not easily attained with catheter ablation alone. This multicenter evaluation confirmed the ability to create linear lesions along the posterior left atrium to generate an efficacy of $80 \%$ of patients in SR at 12 months postprocedure, with an adverse event rate of $11 \%$.

The ability to access the posterior left atrium through a closed-chest epicardial approach enables creation of epicardial lesions under direct visualization. The lesion pattern in the current study did not include isolating the entire posterior left atrium but involved connecting the left to right pulmonary veins in the posterior left atrium parallel to the pericardial reflections. Alternative lesion patterns that isolate the posterior left atrium may be evaluated to compare long-term outcomes. However, heart function must be preserved for any minimally invasive procedure to succeed.

In this evaluation, left atrial size decreased $7 \%$ from baseline, whereas the left ventricular ejection fraction increased $5 \%$ over baseline. These changes are only attributed to the convergent procedure because they were stand-alone treatments of AF. Although not direct measures of atrial contractility, they do represent qualitative improvement in heart function and reverse remodeling.

The success of this completely closed-chest, combined approach in treating persistent and long-standing persistent patients may result from the ability to ablate epicardial substrates, create a comprehensive pattern of continuous 
linear lesions, create lesions along the posterior left atrium, create a cavotricuspid isthmus lesion, and ensure lesion completeness using endocardial mapping and ablation.

The ability to evaluate epicardial lesion integrity addresses the primary technology limitation of all epicardial ablation devices, which are less effective when ablating over fat. By mapping epicardial lesion completeness during the electrophysiologic portion of the procedure, gaps between or along epicardial lesions may be identified and ablated endocardially. In addition, electrophysiologic testing of pulmonary vein isolation and inducibility of atrial arrhythmias provide more definitive metrics of procedure completeness. However, the procedure still depends on catheter ablation techniques, with their documented limitations in creating transmural lesions.

This study was limited by the variability in the timing of the treatment protocol between epicardial and endocardial procedures. Most procedures were performed in a single setting, but because the cohort of patients involved consecutive enrollment, differences between procedure timing and techniques of both epicardial and endocardial ablation may limit poolability for comparative purposes.

The promise of leveraging the positives of 2 medical specialties (cardiac surgery and electrophysiology) has generated interest and is being evaluated for the treatment of persistent and long-standing AF. Multiple hybrid procedures are being evaluated at centers around the world, with varying efficacy and safety results using various epicardial ablation technologies and varying methods to achieve access to the posterior of the heart. ${ }^{8,15,18}$ Procedures are performed as a single procedure on the same day or are staged with a varied time interval between the epicardial ablation and the endocardial ablation. Access to the heart is obtained through bilateral thoracotomies, minithoracotomies, requiring lung deflation and dissection of the attachments between the pericardium and atria. Randomized, controlled, multicenter studies need to be conducted with a standardized lesion pattern and monitoring method with a standardized definition of treatment success.

Outcomes from this 4 European centers' data analysis indicate that the convergent procedure provides a viable treatment option for persistent and long-standing persistent AF patients. Larger studies, potentially controlled and randomized, with a standardized treatment protocol, are needed to definitively establish the impact of this convergent procedure on heart function, cost-effectiveness, and long-term survival.

\section{References}

1. Weerasooriya R, Khairy P, Litalien J, Macle L, Hocini M, Sacher F, et al. Catheter ablation for atrial fibrillation: are results maintained at 5 years of follow-up? J Am Coll Cardiol. 2011;57:160-6.

2. Miyasaka Y, Barnes ME, Gersh BJ, Cha SS, Bailey KR, Abhayaratna WP, et al. Secular trends in incidence of atrial fibrillation in Olmsted County, Minnesota, 1980 to 2000, and implications on the projections for future prevalence. Circulation. 2006;114:119-25.

3. Haïssaguerre M, Jaïs P, Shah DC, Takahashi A, Hocini M, Quiniou G, et al. Spontaneous initiation of atrial fibrillation by ectopic beats originating in the pulmonary veins. $N$ Engl J Med. 1998;339:659-66.

4. Dixit S, Marchlinski FE, Lin D, Callans DJ, Bala R, Riley MP, et al. Randomized ablation strategies for the treatment of persistent atrial fibrillation: RASTA Study. Circ Arrhythm Electrophysiol. 2012;5:287-94

5. Willems S, Klemm H, Rostock T, Brandstrup B, Ventura R, Steven D, et al. Substrate modification combined with pulmonary vein isolation improves outcome of catheter ablation in patients with persistent atrial fibrillation: a prospective randomized comparison. Eur Heart J. 2006;27:2871-8.

6. Roberts-Thomson KC, Stevenson I, Kistler PM, Haqqani HM, Spence SJ, Goldblatt JC, et al. The role of chronic atrial stretch and atrial fibrillation on posterior left atrial wall conduction. Heart Rhythm. 2009;6:1109-17.

7. Kiser AC. Paracardioscopy provides endoscopic visualization of the heart. Innovations. 2009;4:233-5.

8. Gehi AK, Mounsey JP, Pursell I, Landers M, Boyce K, Chung EH, et al. Hybrid epicardial-endocardial ablation using a pericardioscopic technique for the treatment of atrial fibrillation. Heart Rhythm. 2013;10:22-8.

9. Garrett HE, Porter C, Fonger JD, Bravo M, Martin R. Evaluation of an unipolar RF coagulation system for epicardial AF ablation in chronic GLP canine models. J Innov Card Rhythm Man. 2012;3:1042-8.

10. Calkins H, Brugada J, Packer DL, Cappato R, Chen S, Crijns HJG, et al. HRS/ EHRA/ECAS expert consensus statement on catheter and surgical ablation of atrial fibrillation: recommendations for personnel, policy, procedures and follow-up. Heart Rhythm. 2007;4:1-46.

11. Cox JL, Schuessler RB, D'Agostino HJ, Stone CM, Chang B, Cain ME, et al. The surgical treatment of atrial fibrillation, III: development of a definitive surgical procedure. J Thorac Cardiovasc Surg. 1991;101:569-83.

12. Pokushalov E, Romanov A, Corbucci G, Artyomenko S, Turov A, Shirokova N, et al. Use of an implantable monitor to detect arrhythmia recurrences and select patients for early catheter ablation for atrial fibrillation: a pilot study. Circ Arrhythm Electrophysiol. 2011;4:823-31.

13. Martinek M, Aichinger J, Nesser HJ, Ziegler PD, Purerfellner H. New insights into long-term follow-up of atrial fibrillation ablation: full disclosure by an implantable pacemaker device. J Cardiovasc Electrophysiol. 2007;18:818-23.

14. Bertaglia E, Tondo C, De Simone A, Zoppo F, Mantica M, Turco P, et al. Does catheter ablation cure atrial fibrillation? single procedure outcome of drug-refractory atrial fibrillation ablation: a 6-year multicentre experience. Europace. 2010;12:181-7.

15. Weimer T, Bailey MS, Watanabe Y, Marin D, Maniar HS, Schuessler RB, et al. The cox-maze IV procedure for lone atrial fibrillation: a single center experience in 100 consecutive patients. J Interv Card Electrophysiol. 2011;31:47-54.

16. Ad N, Suri RM, Gammie JS, Shenq S, O’Brien SM, Henry L. Surgical ablation of atrial fibrillation trends and outcomes in North America. $J$ Thorac Cardiovasc Surg. 2012;144:1051-60.

17. Boersma L, Castella M, van Boven W, Berruezo A, Yilmaz A, Nadal M, et al. Atrial fibrillation catheter ablation versus surgical ablation treatment (FAST): a 2-center randomized clinical trial. Circulation. 2012;125:23-30.

18. Muneretto C, Bisleri G, Bontempi L, Curnis A. Durable staged hybrid ablation with thoracoscopic and percutaneous approach for treatment of long-standing atrial fibrillation: a 30 month assessment with continuous monitoring. $J$ Thorac Cardiovasc Surg. 2012;144:1460-5 